\title{
L'Importance de la Traduction de la Bible en Langue Chinoise
}

\author{
Eugène Raonimahafaly \\ Université FJKM Raveojaona, Lot Ter 24 H Gilpin Ampandrana, Antananarivo, Madagascar \\ E-mail of the corresponding author: efesianamazava@gmail.com
}

\begin{abstract}
Résumé
La traduction de la Bible en Langue Chinoise fait partie du grand développement de la vie chrétienne en Chine. Le terme technique « importance de la traduction de la Bible en langue chinoise » est utilisé par les spécialistes linguistiques et le bibliciste puisque l'expression « spirituelle chinoise » ne fait pas référence à une dénomination propre; il est transdénominationnel. Dans la Bible chinoise, l'évangile se définit à partir de trois piliers : l'activisme, le biblicisme et le crucicentrisme. L'analyse scientifique de la Bible chinoise améliore l'évangélisation et la théologie chinoise. Au fur et à mesure, la Chine termine la tâche de rédaction du dictionnaire biblique et l'étude étymologique des différents mots techniques chinois en plusieurs tomes. Le comité de rédaction pour la standardisation de texte biblique chinois a élaboré les éditions bibliques chinoises. Ce comité propose un ensemble de symboles phonétiques afin de déterminer les textes, qui fut promulgué en tant qu'alphabet phonétique biblique. Ensuite, l'association de rédacteurs rédige un projet pour la vérification des termes bibliques de la langue chinoise et l'herméneutique. Cette dernière effectue la vérification finale et l'approbation de la terminologie scientifique de la traduction biblique. Finalement, le bureau national d'édition et de traduction eut la déclaration officielle que cette bible chinoise soit utilisée correctement et régulièrement au département missiologique.
\end{abstract}

Mots clés : traduction, Bible, langue, chinoise

DOI: $10.7176 /$ RHSS/11-14-01

Publication date:July $31^{\text {st }} 2021$

\section{Introduction}

La traduction de la Bible en langue chinoise est l'une des moyens efficaces et clés pour l'évangélisation en Chine. Cette traduction une fois créée, a évolué progressivement suivant l'histoire et la situation interculturelle entre la Chine et la plupart des pays chrétiens dans le monde. Contextuellement, Le desservant Flavien ou Gorodecky, qui ne fut autre que le 16ème chef de l'évangélisation ecclésiastique russe entre 1879 et 1883, eut recours à l'intronisation du dialecte chinois au niveau des services ainsi que du fonctionnement de l'actualisation de la transcription du Nouveau Testament en 1884. L'accommodation comporta des notes explicatives concises, dans une forme particulièrement apparentée à la transcription de la terminologie théologique. Par conséquent, Samuel Isaac Joseph Schereschewsky n'avait traduit l'œuvre que partiellement par le biais de la traduction protestante de la Bible lors de la 18ème mission ecclésiastique russe mandatée par Figurovsky ou l'Innocent, qui fut justement l'évêque de Pereyaslav, nommé ultérieurement métropolite de Beijing surtout de la Chine. La réactualisation des ouvrages de Gury fut édictée dans les années 1910 grâce à l'emploi de la langue approximative du vernaculaire chinois.

Dans ce qui suit, nous abordons le contexte linguistique nationalitaire de la traduction de la Bible en langue chinoise. Cette traduction prôna le réaménagement des caractères bibliques chinois diffusés en «putonghua » ou (la « langue commune ») vers 1940. Ces grandes réalisations visaient à jeter les bases de modernisation de la langue et de l'écriture biblique afin de les diffuser à l'ensemble de la population. Cette traduction correspondait à l'idéologie des dirigeants qui voulaient répandre la Bible chinoise auprès du peuple. La simplification des caractères bibliques paraissait nécessaire car elle visait à mettre fin à l'analphabétisme chronique chinoise. Étant donné que l'apprentissage des milliers de caractères de base biblique nécessitait de nombreuses années, la simplification de ces derniers ne pouvait que favoriser l'acquisition de la lecture et de l'écriture de la Bible. On rappelle que, les rédacteurs avaient publié un essai intitulé de la bonne nouvelle, dans lequel ils lançaient un appel pour que la langue écrite de la Bible chinoise soit réformée et simplifiée. Pour les traducteurs bibliques, la simplification des caractères ne devrait être que provisoire. C'est pourquoi l'apprentissage de la Bible en langue chinoise, comme le "putonghua », a été rendu progressivement possible tandis que les langues minoritaires furent «standardisées» en fonction de l'alphabet « pinyin ».

Finalement, l'objet de cette recherche est d'étudier les différentes théories concernant les traductions bibliques en langue chinoise. Ces théories seront exposées en suivant la chronologie et l'évolution du christianisme en Chine. La traduction de la Bible chinoise a été établie de manière scientifique et méthodique pour que ce soit facile à assimiler. Cette traduction a servi épistémologiquement suivant les contextes pour le développement de l'évangélisation en Chine et dans d'autres pays. 


\section{Méthodes}

Le premier type d'analyse qualitative prend en compte l'ordre d'investigation. Il s'agit de soumettre les données à un canevas de questions prédéterminées. Ces questions sont contenues dans le Guide élaboré par Yves SaintArnaud intitulé : " Guide méthodologique de conceptualisation d'un modèle d'intervention ».

Ces méthodes montrent et justifient l'approche systématique qu'on utilise pour effectuer un projet de recherche. Dans tous les cas, les méthodes doivent exposer de façon détaillée et appuyer sur des sources autorisées, les composantes de la méthode adoptée. Cette méthodologie énumère les techniques qu'on compte utiliser dans le cadre de notre recherche. Elles doivent se baser sur les méthodes du projet préliminaire :

- soient par les méthodes générales qui valident la cohérence de l'ensemble du projet de recherche, s'assurant du rapport entre les objectifs poursuivis, la formulation de la question de recherche et les méthodes proposées dans cette recherche ;

- soient par les méthodes spécifiques qui valident l'état de l'art sur le sujet (recherche déjà faite) et conduisent à une formulation dans le terme le plus définitif. Ces méthodes spécifiques assurent les détails techniques de recherche et la planification de recherche. Elles évoquent les principales étapes pour mener à bien la recherche sur le plan théorique. Ces méthodes sont basées sur la lecture de documents ou de livres avec présentation d'une bibliographie bien détaillée.

\section{Résultats}

\subsection{Les ouvrages et traducteurs}

Cette section sur les résultats rapporte les différentes versions bibliques de manière chronologique et les ouvrages des traducteurs bibliques chinois. Les résultats après les ouvrages du professeur Ghazarian se font prolongés spécialement grâce à la diligence du Docteur Joshua Marshman par lequel l'édition du Nouveau Testament se fait proclamée officiellement, c'est-à-dire la valeur ou l'argument statistique de l'édition de la Bible, après des travaux intensifs. Parallèlement, les résultats montrent la variation du taux de production de la traduction de la Bible en langue chinoise. Par conséquent, la statistique de l'évangélisation et l'infrastructure ecclésiale trouveront une croissance parallèle à la variation du taux de production. Les résultats montrent l'évolution vers un taux de croissance positive interne et un taux de répartition positive externe.

Nous trouvons aussi comme résultat l'ouvrage indépendant de Robert Morrison qui a déclaré que si les traducteurs s'entraidaient, les travaux pour la transcription de la Sainte Bible en langue chinoise sous différentes versions auraient été grandement perfectionnés en l'espace d'une vingtaine d'années, identiquement à ce qui s'est passé en une cinquantaine d'années auparavant. Malheureusement, ces deux traducteurs (Robert Morrison et Docteur Joshua Marshman) n'avaient jamais pu comparer ni réviser leurs œuvres respectives. Pourtant, le révérend Josiah Goddard avait quêté auprès du Conseil américain des Missions étrangères un accord pour la révision en totalité de la traduction du Docteur Marshman. Quatorze années après son arrivée sur les terres chinoises, ce dernier avait exposé la version renouvelée du Nouveau Testament.

\subsection{Les caractéristiques de la traduction de la Bible en langue chinoise}

\subsubsection{Les familles linguistiques}

La traduction de la Bible en langue chinoise appartient à huit familles linguistiques. La parenté des langues peut s'établir de la façon qui suit. On compte environ 12411000 locuteurs qui utilisent la Bible chinoise du groupe tibéto-birman; généralement dans les provinces comme Guinan et Guizhou. La famille altaïque comprenait la Bible interprétée en dix-neuf langues qui se subdivisent en trois groupes :

- Le groupe turcique : ouïgour, kazakh, kirghiz, ouzbek, tatar, salar, yugur oriental et touva. On compte 7067000 locuteurs pratiquent la Bible chinoise dans ce groupe.

- Le groupe mongol : mongol, daur, monguor, dongxiang, bao'an et yugur occidental. On dénombre quelque 3172000 locuteurs pour ce groupe.

- Le groupe mandchou-toungouze: mandchou, xibe, hezhen, oroqen et évenki. On compte quelque 47 000 locuteurs pour ce groupe.

Tableau 1. Récapitulation des groupes de la langue chinoise

\begin{tabular}{|c|c|c|}
\hline Groupes & Régions & Locuteurs \\
\hline Groupe turcique & Ouïgour, kazakh, kirghiz, ouzbek, tatar, salar, yugur oriental \\
et touva. & $\begin{array}{c}3172000 \\
\text { locuteurs }\end{array}$ \\
\hline Groupe mongol & Mongol, daur, monguor, dongxiang, bao'an et yugur \\
occoidantal & $\begin{array}{c}3172000 \\
\text { locuteurs }\end{array}$ \\
\hline $\begin{array}{c}\text { Groupe mandchou- } \\
\text { toungouze }\end{array}$ & Mandchou, xibe, hezhen, oroqen et évenki & 47000 locuteurs \\
\hline
\end{tabular}

3.2.2 L'écriture et la traduction de la Bible en langue chinoise

Il existe différents systèmes de transcription de la Bible en langue chinoise. On connaît le fameux système 
idéographique chinois, qui fait le malheur des Occidentaux. Quand on Occidental dit «que c'est du chinois», c'est qu'il n'y a rien à comprendre ; on parle aussi de chinoiseries, c'est-à-dire de « complication inutile et extravagante ». Parallèlement à ce système, on mentionne l'alphabet pinyin dans la Bible chinoise (alphabet latin) utilisé en Chine, l'alphabet « Bopomofo », non latinisé, employé à Taiwan, l'alphabet « penkyamp » inspirée du pinyin, mais adapté au cantonais, le système latinisé dit de Wade-Giles (pour le mandarin) ; ce dernier s'est développé d'après un système créé par Thomas Wade au milieu du XIXe siècle ; il a été le principal système de transcription dans le monde anglophone pendant la majorité du XXe siècle. L'alphabet Wade-Giles utilise des traits d'union pour séparer toutes les syllabes (par exemple, Hsi-an et Hsi-fan), alors que le pinyin ne sépare que les syllabes ambiguës, avec une apostrophe (par exemple, Xi'an, mais Xifan).

3.2.3 L'alphabet pinyin

Le gouvernement chinois a introduit un alphabet appelé « pinyin ». Le président Mao Tsé-toung avait entrepris cette gigantesque réforme linguistique au but de faciliter l'alphabétisation des masses, de favoriser l'apprentissage du chinois chez les jeunes enfants, de créer des langues écrites pour les minorités et de permettre l'informatisation des banques de données. Les traducteurs de la Bible en langue chinoise ont été profités cette politique chinoise et ils assument le droit d'auteur pour conserver toutes les éditions de la Bible chinoise. L'objectif de ces traducteurs est de poursuivre la romanisation de l'écriture de la Bible (l'alphabet pinyin étant calqué pour l'essentiel sur l'alphabet latin) et d'orienter ces traductions de la Bible en langue chinoise existante vers l'alphabet pinyin. L'alphabet dit : « Hanyu Pinyin » comporte 30 formes qui permettent de représenter 26 lettres de l'alphabet latin. Les Bibles chinoises ont été déclarées officiellement au sein de l'Eglise internationale et elles ont été adoptée au Projet de transcription phonétique de la langue chinoise le « pinyin ».

Les traducteurs de la Bible en langue chinoise ont été travaillé aussi aux différentes activités au tour de l'Eglise chinoise telles que :

- $\quad$ prononciations des noms de l'Eglise en pinyin

- $\quad$ méthode de rectification des termes liturgiques en pinyin

- $\quad$ principes de base pour les méthodes de rectification des mots théologiques en pinyin

- $\quad$ transcription en pinyin des cantiques chinois.

- $\quad$ méthode de transcription en pinyin des noms de personnes en chinois aux sacrements.

- $\quad$ règle de transcription en pinyin les noms de lieux ecclésiaux en chinois.

- $\quad$ système d'alphabet de la Bible chinoise pour les sourds

- braille chinois

\section{Discussion}

Les suggestions ont fourni le développement d'évangélisation dans le monde entier surtout en Chine grâce à l'ouverture, aux réformes et à l'intégration. L'accès de la traduction biblique en langue chinoise est indispensable pour l'amplification de la vie chrétienne en Chine, mais cette entrée signifie aussi le renforcement de développement social et culturel chinois. Cela veut dire qu'avec le renforcement de l'évangélisation chinoise, les lacunes de la vie chrétienne chinoise vont émerger, en particulier en ce qui concerne la réduction de l'écart régional de traduction de la Bible en langue chinoise après les résultats acquis. Ainsi, les associations de traducteurs doivent exercer leurs fonctions d'ajustement méthodique et efficace pour la bonne coordination des développements des différentes régions.

D'après ces résultats, le développement de la traduction repartie en deux étapes successives. Les administrations de traducteur doivent renforcer ses stratégies dans les régions retardées. Une des raisons principales du retard de certaines régions est leur manque de communications. Par conséquent, afin d'assurer la réduction de la disparité, ces régions ont besoin de ces aides. L'administration doit ainsi ajuster ses parts de stratégie en inclinant plus vers les régions retardées. En même temps, ces aides doivent être orientés vers l'amélioration des infrastructures, des structures sectorielles, culturelles et de la préservation de l'environnement chinois. Cependant, en dehors des stratégies spirituelles, ce centre administratif doit renforcer ses aides financières et encourager ainsi les investissements ou les collectes des fonds indirects. Par exemple, le centre administratif peut autoriser des moyens plus avantageux pour les régions retardées telles que des taux plus attractifs au niveau matériaux et financiers. De plus, ce centre peut émettre, par exemple, de bons spéciaux pour le développement régional en Chine afin de pallier le manque d'emprunts à long terme de ces régions. Le centre de traduction chrétienne peut aider les régions retardées ainsi que les délégations de ces régions à être cotées en bourse pour avoir plus de capitaux de développement de traduction de la Bible. L'association chrétienne peut autoriser des ouvertures plus larges dans les régions retardées.

\section{Réferences}

Aristote, Ethique à Nicomaque, traduit en chinois par Deng Anqing, Presse du Peuple, Pékin, 2010, ISBN 9787010092164

Aristote, Ethique à Nicomaque, traduit en chinois par Liao Shenbai, Presse de la Commerce, Shanghai, 2013, 
ISBN 7100035759

Aristote, La Politique, translation en chinois par Wu Shoupeng, Presse de la Commerce, Shanghai, 1965, ISBN 9787100018036.

Armstrong, N. (2005), Translation, linguitics, culture: a French-English hanbook, Multilingual Matters, New York, ISBN 1853598054

Avramescu, C. (2011), An Intellectual History of Cannibalism, traduit en anglais par Alistair Ian Blyth, Princeton University Press, ISBN-10: 0691152195, ISBN-13: 978- 0691152196,

Baeck, L. (2002), L'Essence du judaisme, translation en chinois FU Yongjun, YU Jian, Presse de l'Université de Shandong, Shangdong, ISBN 7560722393, 9787560722399,

Baker, M. (2011), Translation and Conflict, a Narrative Account, traduit en chinois par Zhao Wenjing, Presse de l’Université de Pékin, Pékin, ISBN 9787301191392

Bakhtine, M. M. (1998), Le Texte, le dialogisme et l'humanité, traduction en chinois par Bai Chunren et Xiao He, Presse de l'Instruction, Hebei, ISBN 9787543431256 ,

Barthes, R. G. (2009), Le Plaisir du texte, interprète en chinois par Tu Youxiang, Presse du Peuple de Shanghai, Shanghai, ISBN 9787208085862

La Bible Disponible sur : Groupe Épiscopale Liturgique pour les Francophones. 20 juillet 2014.

Yu, J. (2009), The Ethics of Confucius and Aristotle: Mirrors of virtue, traduction en chinois par Lin Hang, Press de l’Université du Peuple Chinois, Pékin, ISBN 9787300103051 / B620.

Yu L. (2006), La Révolution de la diffusion de la foi et la naissance des Églises en Chine, Presse des Olives, Taiwan, 957413587X, 9789574135875

Zheng L. (2011), les missionnaires dans l'histoire actuelle de la Chine, Presse des Littératures innovantes, Taiwan, ISBN 9789868681583.

Shang, B. (2002), Vérification sur les traditions folkloriques sociales de toutes les races de l'histoire, Bibliothèque de Guangling, Jiangsu, ISBN 9787806431610 ,

Shun, Y. (1989), Le passé et le présent du catholicisme en Chine, Presse des Sciences Sociales de Shanghai, Shanghai, ISBN 9787805153261 , pages $146-152$.

Tracy, D. (1998), Plurality and Ambiguity, translation en chinois par Feng Chuan, Presse Jointe de SDX, Shanghai, ISBN 9787542610294 